\title{
Ability of clinical isolates of group A streptococci to adhere to and invade HEp-2 epithelial cells
}

\author{
V. R. BENNETT-WOOD, J. R. CARAPETIS* and R. M. ROBINS-BROWNE \\ Microbiological Research Unit, Department of Microbiology and Infectious Diseases, Royal Children's Hospital \\ and Department of Microbiology and Immunology, University of Melbourne, Parkville, Victoria 3052, Australia
}

\begin{abstract}
Individual strains of group A streptococci (GAS) differ in virulence, but the reasons for these differences are incompletely understood. To determine if the ability of GAS to cause invasive disease corresponded with their capacity to adhere to or invade epithelial cells, 63 clinical isolates of GAS (40 from patients with systemic infection and 23 from superficial disease) were examined in quantitative assays of bacterial adhesion to and invasion of HEp-2 cells, a continuous line of human pharyngeal epithelial cells. The results showed that individual isolates of GAS varied considerably in their ability to adhere to and penetrate HEp-2 cells. However, on the whole, strains from patients with invasive disease adhered to cells in numbers $c .1 .5$ greater than those from superficial infection. Paradoxically, strains from patients with invasive disease invaded HEp-2 cells to a significantly lesser extent than those from superficial sites, with a two-fold difference in invasion index (defined as the percentage of cell-associated bacteria located intracellularly). To determine if these differences were caused by differences in the production of hyaluronic acid capsule or $M$ protein by the two groups of bacteria, the adherence and invasive capacities of bacteria carrying defined mutations in the genes for these factors were examined. Although M18-protein-deficient bacteria were less adherent to HEp-2 cells than the wild-type, neither the hyaluronic acid capsule nor the $M$ protein had a significant influence on the ability of GAS to adhere to or invade HEp-2 cells. The results of this study demonstrate that there are biological differences between GAS isolates associated with invasive and superficial diseases and that these differences can be demonstrated by an assay of bacterial adherence to and invasion of HEp-2 epithelial cells.
\end{abstract}

\section{Introduction}

Streptococcus pyogenes (group A streptococcus; GAS) is a frequent and versatile cause of human disease. During the past decade, there has been a world-wide increase in the incidence of invasive streptococcal infections, notably cellulitis and necrotising fasciitis, and the emergence of a streptococcal toxic shock syndrome $[1,2]$. The reasons for the upsurge in these diseases are not known, although they may be explained in part by the spread of highly virulent subclones of GAS. Thus, many serious infections with GAS are caused by strains of M-protein types 1 or 3 [3]. Although specific virulence factors associated with increased virulence of GAS have not been identified,

Received 6 Jan. 1998; accepted 5 Feb. 1998.

Corresponding author: Professor R. M. Robins-Browne.

* Present address: Menzies School of Health Research, PO Box 41096, Casuarina NT 0811, Australia. some strains obtained from patients with invasive disease in the Northern hemisphere have a mucoid phenotype $[4,5]$, indicating the presence of large amounts of hyaluronic acid capsular material on the bacterial surface. In addition, many highly virulent strains carry the speA gene, which encodes production of streptococcal pyrogenic exotoxin A $[1,3,6]$, although recent work suggests that particular genotypic or phenotypic characteristics - including the presence of speA - are not common to all invasive isolates [7].

GAS are generally regarded as extracellular pathogens that cause disease by spreading through tissue matrices and secreting potent protein exotoxins, whilst impeding phagocytosis. However, several groups of workers have shown recently that GAS can penetrate epithelial cells in vitro [8-10]. For example, La Penta et al. [9] reported that strains of M-type 1 GAS associated with blood-stream infections are able to invade epithelial cells more efficiently than strains 
from patients with uncomplicated pharyngitis, suggesting that the ability to penetrate the respiratory epithelium may contribute to the virulence of these bacteria. Invasion of epithelial cells by GAS requires an intact host cell cytoskeleton [8], and appears to be mediated by an interaction between $S$. pyogenes fibronectin-binding protein $(\mathrm{SfbI})$ and fibronectin on host cells [11].

A 12-year retrospective analysis of bacteraemia caused by GAS at the Royal Children's Hospital, Melbourne, confirmed the association of M-type 1 bacteria carrying the speA $^{+}$gene with invasive disease [12]. It also found that isolates that could not be M-typed (M-non-typable, MNT) were the most common GAS overall and the second most common variety obtained from patients with invasive disease. One particular mucoid, MNT, speA ${ }^{+}$strain with a distinctive DNA fingerprint was associated with toxic shock syndrome and septic shock in two children, one of whom died [12]. The aim of the present study was to compare the virulence-associated characteristics of isolates of GAS from patients with invasive and non-invasive infections, mainly in a tissue culture model for bacterial adhesion and invasion. The study also examined the contribution of the hyaluronic acid capsule and $\mathrm{M}$ protein to the behaviour of G $\% \mathrm{~S}$ in this assay.

\section{Materials and methods}

\section{Bacteria}

The bacteria examined in this study have been reported previously [12]. They included 63 clinical isolates obtained between 1986 and 1993 from adult and paediatric patients attending hospitals within $60 \mathrm{~km}$ of Melbourne. For the purposes of this study, 40 isolates of GAS cultured from blood, cerebrospinal fluid, joint fluid or bursa fluid were regarded as invasive, whilst 23 isolates from patients with pharyngitis or superficial skin infections were considered non-invasive. Five isolates (four from patients with invasive disease and one from non-invasive disease) displayed a highly mucoid phenotype when grown on solid media. Serotyping of isolates was performed at the Streptococcus Reference Laboratory, Communicable Disease Centre, Porirua, New Zealand [12]. Overall, 25 isolates were MNT (12 invasive, 13 non-invasive); 20 were M1 (15 invasive, 5 non-invasive); seven were M12 (all invasive) and 11 isolates belonged to other M-types.

Defined mutants and their matched parent strains were used to assess the contribution of the hyaluronic acid capsule and $M$ protein to the adhesive and invasive ability of these bacteria. Strain 282S1 (M-type 18) is a spontaneous streptomycin-resistant derivative of mucoid strain 87-282 which was originally cultured from the pharynx of a child with rheumatic fever [13]. Strain TX4 is a non-mucoid derivative of strain 87282 obtained by mutagenesis with the conjugative transposon, Tn916. TX4 also has a 9.5-kb chromosomal deletion adjacent to the site of insertion of Tn916, which resulted in the loss of two enzymes involved in hyaluronic acid biosynthesis: has $\mathrm{A}$, encoding hyaluronate synthase and has $\mathrm{B}$, encoding UDP-glucose dehydrogenase $[13,14]$. S. pyogenes JRS4 (M-type 6) is a spontaneous streptomycinresistant derivative of strain D471 [15]. JRS75 is an $\mathrm{M}^{-}$Tn916 mutant of JRS4 derived by mutagenesis of the emm6.1 gene $[15,16]$.

\section{Bacterial adhesion to and invasion of epithelial cells}

The ability of GAS to adhere to and invade HEp-2 cells (a continuous line of epithelial cells derived from human pharyngeal carcinoma) was assessed in a quantitative assay as described previously, with some modifications $[9,17]$. The conditions used for these assays were essentially the same as those described by La Penta et al. [9] and were verified in a series of preliminary experiments to examine the influence of the following parameters on the validity and reproducibility of the findings: degree of confluency of the HEp-2 cell monolayer, the length of time for which bacteria were incubated with the HEp-2 cells (1.5-4 h), the number of bacteria added to the monolayer $\left(10^{6}-\right.$ $10^{9} \mathrm{cfu}$ ) and the concentration of gentamicin used to eliminate extracellular bacteria $(50-200 \mu \mathrm{g} / \mathrm{ml})$.

For the assay, bacteria were grown in Brain Heart Infusion Broth (BHIB; Oxoid, Basingstoke), without shaking at $37^{\circ} \mathrm{C}$ for $c .6 \mathrm{~h}$ and then transferred into fresh BHIB for overnight incubation. After washing and resuspension in sterile phosphate-buffered saline (PBS), $\mathrm{pH} 7.4$, c. $10^{7} \mathrm{cfu}$ were added to each well of a 24-well tissue culture tray (Nunc, Roskilde, Denmark) containing a confluent monolayer of HEp-2 epithelial cells. Bacteria were incubated with these cells in tissue culture medium (comprising minimal Eagle's medium with fetal calf serum 5\%, D+ mannose $0.5 \%$ and $10 \mathrm{mM} \mathrm{N}$-2-hydroxyethylpiperazine-N'-2-ethansulphonic acid (HEPES; Sigma), for $2 \mathrm{~h}$ at $37^{\circ} \mathrm{C}$. Non-adherent bacteria were then removed by washing each well three times with PBS. The total number of bacteria that remained cell-associated (i.e., bound to the cell surface and internalised) was determined by lysing the HEp-2 cells in $0.2 \mathrm{ml}$ of PBS containing digitonin (Sigma) $0.1 \% \mathrm{w} / \mathrm{v}$. The resultant suspension was diluted in BHIB and the number of viable bacteria $/ \mathrm{ml}$ was determined on duplicate horse blood agar plates. Given that the majority $(50-99 \%)$ of bacteria were located extracellularly (see below) this value was taken as an indication of bacterial adhesion.

The assay for bacterial invasive capacity was similar to that for adhesion, except that after removal of nonadherent bacteria by washing, cells were incubated for a further $2 \mathrm{~h}$ at $37^{\circ} \mathrm{C}$ in tissue culture medium 
containing gentamicin $100 \mu \mathrm{g} / \mathrm{ml}$ and penicillin $\mathrm{G}$ $5 \mu \mathrm{g} / \mathrm{ml}$ to kill any remaining adherent extracellular bacteria. After washing with PBS, HEp-2 cells were then lysed with digitonin and the number of viable bacteria in each well was determined as described above. The data were used to derive an invasion index, which was defined as the percentage of cell-associated bacteria that were recovered in the invasion assay [17].

To ensure that the formation of aggregates or chains by GAS did not give rise to falsely low viable counts in the adhesion or invasion assays, a number of the samples for determination of viable counts were divided into two portions, one of which was sonicated for $15 \mathrm{~s}$. Although this treatment effectively disrupted bacterial aggregates and chains as determined by light microscopy, the numbers of viable bacteria recovered from sonicated and unsonicated preparations were almost identical.

\section{Electron microscopy}

Scanning and transmission electron microscopy were performed to visualise bacterial adherence to and invasion of HEp- 2 cells by GAS. For scanning electron microscopy, a c. $80 \%$ confluent monolayer of HEp-2 cells in 24-mm diameter plastic trays (Nunc) containing 12-mm diameter glass coverslips was incubated for $3 \mathrm{~h}$ with $10^{7} \mathrm{cfu}$. Non-adherent bacteria were removed by washing three times with PBS. Cells were then fixed with glutaraldehyde $2.5 \%$ for $30 \mathrm{~min}$, post-fixed in osmium tetroxide for $15 \mathrm{~min}$, and dehydrated through a graded series of ethylene glycol (BDH Chemicals, Kilsyth, Victoria, Australia) and 2-ethoxyethanol (Fisons, Homebush, NSW, Australia). They were then subjected to critical point drying and coated with gold with an Edwards S150B sputter coater, before viewing in a Phillips 515 scanning electron microscope at an accelerating voltage of $20 \mathrm{kV}$.

For transmission electron microscopy, HEp-2 cells were grown to $c$. $80 \%$ confluency in $50-\mathrm{mm}$ diameter plastic Petri dishes (Becton Dickinson, Plymouth), after which $10^{8} \mathrm{cfu}$ were added and incubated with the tissue culture cells for $3 \mathrm{~h}$ at $37^{\circ} \mathrm{C}$. Wells were then washed three times with PBS to remove nonadherent bacteria and fixed in glutaraldehyde $2.5 \%$ for $1 \mathrm{~h}$. After further washing, the cell monolayer was removed. Cells were then centrifuged to form a pellet, which was post-fixed in osmium tetroxide, dehydrated through a graded acetone series and embedded in epon-araldite epoxy resin. Thin sections were cut and stained with uranyl acetate and lead citrate before viewing with a Phillips 400 electron microscope at $60 \mathrm{kV}$.

\section{Examination for $M$ protein}

Bacteria were assayed for the presence of $\mathrm{M}$ protein by the human whole blood bactericidal assay, which relies upon the ability of M-protein-producing strains of GAS to resist phagocytosis and subsequent destruction by polymorphonuclear leucocytes [18]. The only variation from the original method was that bacteria were rotated in blood at $4 \mathrm{rpm}$ rather than at $8 \mathrm{rpm}$. Results of the assay were expressed as a growth index, defined as $\log _{2}$ of the change in colony count after $3 \mathrm{~h}$. Because of the slower rotation speed used in the assay and the consequent slower growth, a growth index of $>1$, rather than $>6$, was defined as an indication of the presence of $M$ protein [18]. The validity of this modification was established with control strains JRS4 $\left(\mathrm{M}^{+}\right)$and JRS75 $\left(\mathrm{M}^{-}\right)$. When the growth index of a particular strain was $<1$, the assay was repeated with blood from a different donor to ensure that poor growth was not due to type-specific antibodies in the donor's plasma. Blood from up to eight different donors (including two 6-year-old children) was tested before a strain was classified as $\mathrm{M}$-protein-negative $\left(\mathrm{M}^{-}\right)$.

\section{Statistical analysis}

Bacterial counts obtained from at least three separate adhesion and invasion assays (performed in duplicate) were subjected to $\log$ transformation to ensure that the data were normally distributed. Data were then analysed by Student's two-tailed $t$ test. A critical $\mathrm{p}$ value of 0.05 was used for all analyses.

\section{Results}

\section{Overall assessment of the adhesive and invasive capacity of $G A S$}

Individual isolates of GAS varied considerably in their ability to adhere to and invade HEp-2 cells, but the relative levels of adhesion and invasiveness were similar in repeat experiments. The number of bacteria recovered in adherence assays ranged from $c .20$ to $800 \%$ of the original inoculum; those recovered from invasion assays ranged between c. 1 and $80 \%$ of the inoculum. Values in excess of $100 \%$ in adherence assays indicated that the bacteria had proliferated during the course of the assay, but examination of bacterial growth in tissue culture medium in the absence of HEp-2 cells indicated that there was little variation in the growth rates of individual isolates (data not shown).

The variability in the adhesive and invasive capacity of individual GAS isolates was confirmed by electron microscopy, which showed that isolates that gave elevated values in adhesion assays were located on the epithelial cell surface (Fig. 1), whereas those that gave high counts in invasion assays were located predominantly within intracytoplasmic vacuoles (Fig. 2).

\section{Characteristics of isolates from patients with invasive and non-invasive disease}

Isolates from patients with invasive disease adhered to HEp-2 cells in significantly greater numbers (mean 215 

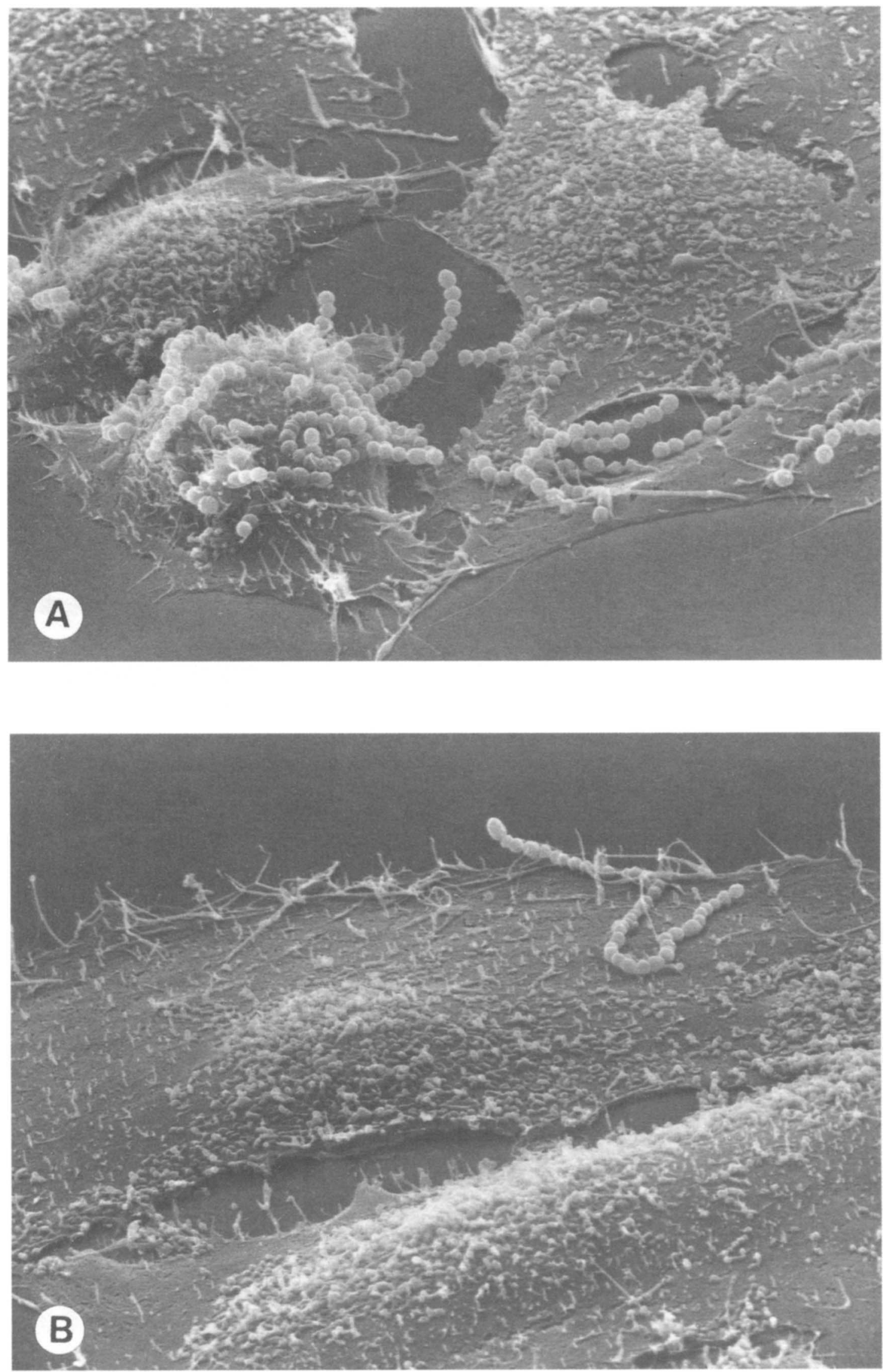

Fig. 1. Scanning electron micrograph of a HEp-2 epithelial cell monolayer incubated for $3 \mathrm{~h}$ with $\mathbf{A}$, a strongly adherent or B, a moderately adherent isolate of GAS originally obtained from blood cultures. In a quantitative adherence assay of bacterial adherence to HEp-2 cells, $248 \%$ of an inoculum containing isolate A was retrieved from HEp- 2 cells after $3 \mathrm{~h}$, compared with $49 \%$ of an equivalent inoculum of the isolate shown in B. Magnification $\times 4850$ 

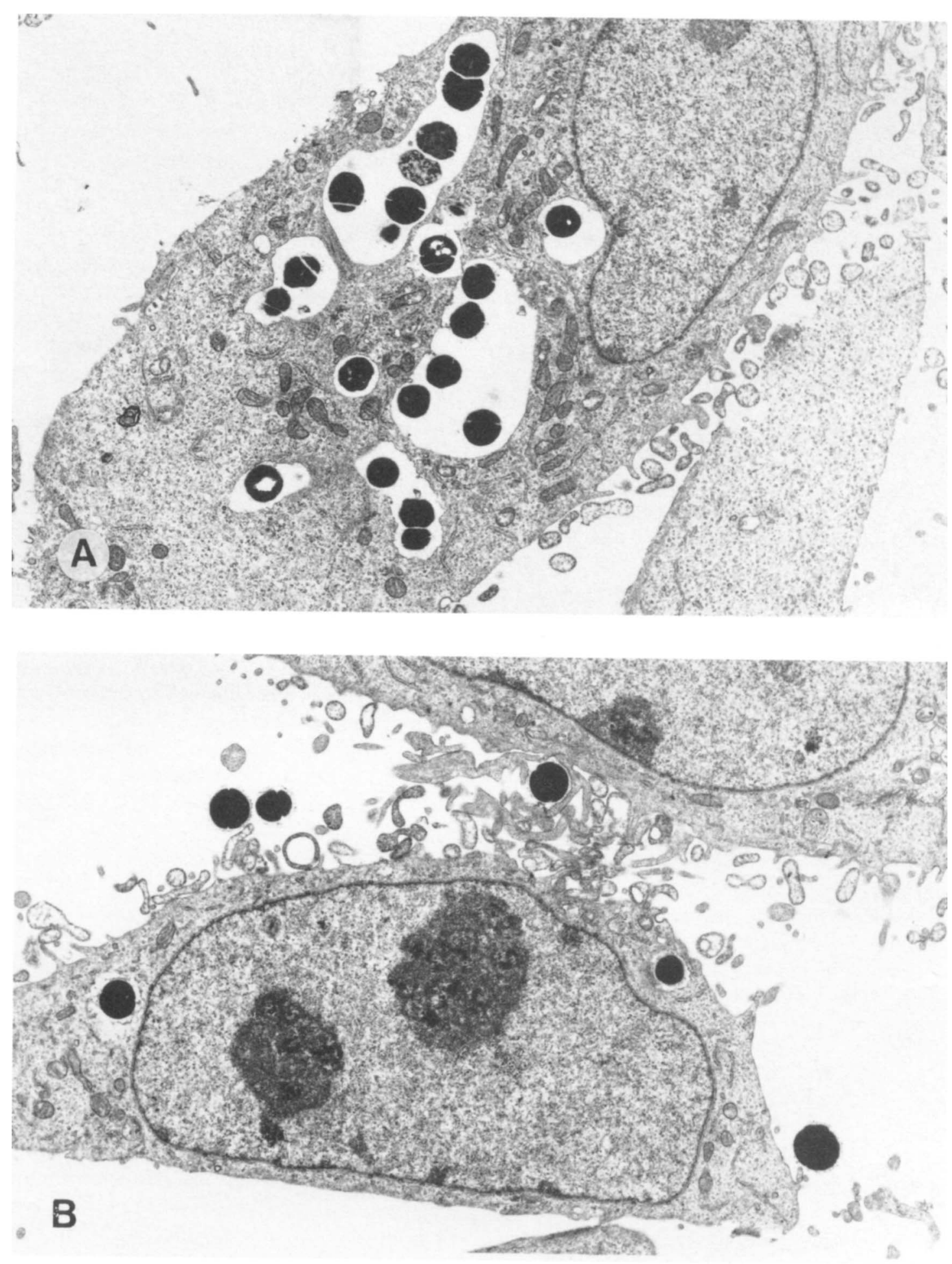

Fig. 2. Transmission electron micrograph of HEp-2 epithelial cells incubated for $3 \mathrm{~h}$ with isolates of GAS from blood cultures: A, a mucoid invasive strain; B, a non-mucoid, weakly invasive strain. Note the bacteria contained within large cytoplasmic vacuoles in A, reflecting the large amount of capsular material. Magnification $\times 7280$.

SD $117 \%$ of the original inoculum recovered from the tissue culture assay) compared with those associated with superficial infections (143 SD $100 \% ; \mathrm{p}=0.04$, Student's two-tailed $t$ test). Paradoxically, bacteria from patients with invasive disease were significantly less invasive, with an invasion index of 17.1 SD $16.8 \%$ compared with those from patients with superficial infections (36.6 SD 21.8\%; p < 0.001).

\section{Influence of hyaluronic acid capsule and $M$ protein}

GAS strains associated with severe invasive disease may exhibit a mucoid phenotype indicating the presence of large quantities of capsular material [6]. To determine whether or not the capsule influenced the interaction of the bacteria with HEp-2 cells, the adherence and invasion assays were repeated with a 
well-characterised mucoid strain of GAS $(282 \mathrm{~S} 1)$ and its decapsulate derivative (TX4) $[13,14]$. The results indicated that the presence of the capsule did not affect the ability of strain $282 \mathrm{~S} 1$ to associate with or penetrate HEp-2 cells (Table 1).

In view of the large number of MNT isolates of GAS obtained from patients with invasive disease [12], these isolates were characterised further. The results showed that 10 of $12 \mathrm{MNT}$ isolates associated with invasive disease expressed $\mathrm{M}$ protein, as demonstrated by their resistance to phagocytosis in the whole blood bactericidal assay [18]. However, two isolates that were associated with life-threatening infections [12], yielded a growth index of 0 (i.e., no viable bacteria recovered) in blood obtained from eight separate individuals, suggesting that these bacteria did not express $M$ protein under the conditions used in this study. These two isolates evidently belonged to the same clone as shown by molecular typing with random amplification of polymorphic DNA (RAPD type F) [12].

In-vitro studies to evaluate the contribution of $M$ protein to GAS adhesion to epithelial cells have yielded conflicting results $[15,19,20]$. This study investigated the role of $M$ protein by performing adherence and invasion assays with an M-type 6 strain, JRS4, and its $\mathrm{M}^{-}$derivative, JRS75. The results showed that JRS4 $\left(\mathrm{M}^{+}\right)$adhered almost twice as avidly as JRS75 $\left(\mathrm{M}^{-}\right)$, but this difference did not reach statistical significance (mean difference 31\%, $95 \%$ confidence interval $3.9-65.9 ; \mathrm{p}=0.08$, Student's $t$ test, two-tailed; Table 1). However, the invasion index obtained with these two strains was almost identical (mean difference $=1.4 \% ; 95 \%$ confidence interval 19.2-22.0), indicating that the M18 protein of JRS4 does not influence the invasive capacity of this strain.

\section{Discussion}

Although GAS colonises the upper respiratory tract mucosa and sometimes passes through the respiratory epithelium to cause invasive disease, knowledge of the bacterial components that facilitate these processes is incomplete [3]. Candidates for GAS colonisation factors include lipoteichoic acid, hyaluronic acid, $M$ protein and $S$. pyogenes fibronectin-binding protein $[1,3]$. $M$ protein and fibronectin-binding protein may also contribute to the ability of GAS to invade epithelial cells $[1,11]$.

This study used a tissue culture assay to compare GAS isolates from patients with systemic and superficial infection to determine if apparent differences in bacterial virulence were reflected by different behaviour in vitro. The bacteria used for these studies were obtained over a 12-year period and included a variety of M-types, as well as many MNT strains, and, therefore, were not clonal. This may have accounted for the considerable variation in the data obtained with individual isolates in the assays of adhesion to and invasion of tissue culture cells. However, in general isolates from patients with invasive disease adhered to HEp-2 cells, a continuous line of human pharyngeal epithelial cells, in significantly greater numbers than those from superficial infections $(p=0.04)$. However, paradoxically, the systemic isolates were significantly less invasive than the other strains $(p<0.001)$. These findings are in distinct contrast to those reported with group B streptococci, in which isolates from patients with systemic infection were found to be significantly more invasive than those associated with superficial disease [21].

The results of the present study indicated that the factors that mediated the adhesion of GAS to HEp-2 cells differed from those involved in invasion, and furthermore, there were clear biological differences between GAS isolates associated with systemic and superficial infections. The increased adhesion of more virulent strains to epithelial cells may indicate the presence of novel adhesins in these bacteria or their enhanced expression of recognised adhesins. Reasons for the paradoxically reduced in-vitro invasiveness of these strains are less clear. It is possible that bacterial strains associated with systemic infection produce surface antigens or toxins that interfere with the ability of HEp-2 cells to ingest them, e.g., by affecting the integrity of the cytoskeleton. However, light and

Table 1. Influence of the hyaluronic acid capsule and $M$ protein on the ability of group A streptococci to adhere to and invade HEp-2 epithelial cells

\begin{tabular}{|c|c|c|c|}
\hline Strain & Characteristics & Cell-associated bacteria* & Invasion index ${ }^{\dagger}$ \\
\hline 282S1 & Streptomycin-resistant mutant of wild-type capsulate strain, $87-282$ & 165 SD $136(12)$ & $6.4 \mathrm{SD} 4.2(12)$ \\
\hline TX4 & Non-mucoid, decapsulate Tn916 mutant of $87-282$ & $199 \mathrm{SD} 132(12)^{*}$ & $11.4 \mathrm{SD} 8.7(11)^{\ddagger}$ \\
\hline JRS4 & Streptomycin-resistant mutant wild-type M6 strain, D471 & 75 SD $47(10)$ & 43.3 SD $21.9(10)$ \\
\hline JRS75 & M-protein-negative derivative of JRS4 (emm6.1::Tn916) & 44 SD $31(12)^{\S}$ & $44.7 \mathrm{SD} 24.0(12)^{\mathrm{s}}$ \\
\hline
\end{tabular}

*Values are the mean and SD (number of experiments) of the percentage of the original bacterial inoculum recovered from HEp- 2 cells after $3 \mathrm{~h}$.

tValues are the mean and SD (number of experiments) of the percentage of cell-associated bacteria that were internalised.

Not significantly different from capsulate strain, 282S1 ( $p \geqslant 0.1$, Student's two-tailed $t$ test).

${ }^{\S}$ Not significantly different from M-protein-bearing strain, JRS4 ( $\mathrm{p} \geqslant 0.1$, Student's two-tailed $t$ test). 
electron microscopic examinations of cells incubated with virulent GAS did not reveal any evidence of cytotoxicity. Another possible explanation is that virulent GAS penetrate epithelia by passing between the cells rather than through them, whereas less virulent strains colonise the pharynx in part by entering and persisting within pharyngeal epithelial cells [10]. As the assay used here (and in other studies of streptococcal invasion) specifically selected against extracellular bacteria, those with the capacity to pass between epithelial cells would be counter-selected. This hypothesis could be tested by performing invasion studies with polarised epithelial cell monolayers.

Wessels and Bronze [14] have shown that the hyaluronic acid capsule contributes to the ability of GAS to become established on the respiratory mucosa of mice. However, the present study found that the adhesive and invasive ability of TX4, a decapsulate mutant, did not differ significantly from that of its parent strain. As the role of the capsule as an adhesin in human infections is uncertain, it is not clear if the discrepancy reflects limitations of the animal or the tissue culture model.

Experimental data regarding the contribution of $M$ protein to GAS adhesion are conflicting. In this study, quantitative analysis of adherence of $S$. pyogenes JRS75, an $\mathrm{M}^{-}$mutant, indicated that $\mathrm{M}$ protein may contribute to the ability of GAS to adhere to HEp-2 cells (Table 1). This confirms the observations of some workers [20], but contradicts those of others $[15,19]$. The reasons for these differences are unknown, although they may reflect variations in the methods used to cultivate the bacteria for the in-vitro adherence assays, and the methods used to quantify bacterial adherence [20].

The major contribution of $M$ protein to streptococcal virulence is to mediate increased resistance to phagocytosis by polymorphonuclear leucocytes [22]. Many clinical isolates of GAS are MNT $[6,12,18,23]$, suggesting that they may lack $M$ protein, but the usual explanation is that these strains express variants of $\mathrm{M}$ protein for which there are currently no standardised antisera [18]. Tran et al. [18] developed a whole blood bactericidal assay to demonstrate the presence of $M$ protein on MNT strains of GAS obtained from healthy schoolchildren. The assay relies upon the anti-phagocytic properties of $\mathrm{M}$ protein, but may produce misleading results if the blood donor has circulating antibodies to the $M$ protein of the test strain, because these antibodies may opsonise the bacteria, allowing them to be phagocytosed despite their carriage of $\mathrm{M}$ protein. For this reason Tran et al. [18] advocated the reexamination of isolates that yield low growth indices with blood from a different donor. The present study identified two MNT isolates of GAS, evidently representatives of the same clone, which were associated with severe invasive disease, but yielded a low growth index in blood obtained from eight different donors, and hence appeared to lack $M$ protein. The reasons for the apparent absence of $M$ protein from these bacteria are unknown. Given that both strains were originally obtained from the blood of previously healthy children and, therefore, were evidently able to resist phagocytosis, a possible explanation is that these bacteria did not produce $M$ protein under the conditions used for the assays in the present study. However, recent findings suggest that, under experimental conditions, some strains of GAS can resist phagocytosis in the absence of $M$ protein and that the hyaluronic acid capsule may provide some of this resistance [24]. It is possible that the two MNT isolates described in the present study are invivo examples of this in-vitro phenomenon. One of our current lines of research is to investigate this question further.

In conclusion, this study has confirmed and extended the findings of La Penta et al. [9] by showing that most clinical isolates of GAS are able to penetrate epithelial cells of respiratory origin. The clinical relevance of this finding was not immediately apparent, because strains from patients with invasive infections were overall significantly less invasive in vitro than those associated with superficial disease. This discrepancy may indicate the inappropriateness of investigating the invasive properties of GAS by a tissue culture assay. Nevertheless, the assay did indicate significant biological differences between the two groups of bacteria examined in this study, which were not evident from assays of conventional virulence determinants [12]. To date, most studies of GAS virulence have focused on the substances produced by, receptors located on, or genes present in these bacteria [1-4]. These investigations have revealed some differences between highly virulent and less virulent strains, but have failed to provide a clear indication of any specific factor or factors associated with highly invasive strains, in that different investigators have found different virulence determinants to be important in this regard. The approach taken in the present study was to examine the behaviour of GAS from various clinical sources in an in-vitro assay which may reflect the early stages of GAS infection of their hosts. The differences found between GAS associated with systemic disease and those from patients with superficial infections may reflect inherent differences in virulence between these two groups of bacteria. The fact that these differences are not associated with any known bacterial virulence factor suggests that they are worthy of further investigation.

We are indebted to D. Martin, Streptococcus Reference Laboratory, Communicable Disease Centre, Porirua, New Zealand, for serotyping the clinical isolates used in this study. We gratefully acknowledge the generosity of M. Wessels, Harvard Medical School, Boston, MA USA for the gift of group A streptococcal strains 282S1and TX4, and 
J. R. Scott, Emory University Health Center, Atlanta, GA, USA for strains JRS4 and JRS75.

\section{References}

1. Curtis N. Invasive group A streptococcal infection. Curr Opin Infect Dis 1996; 9: 191-202.

2. Stevens DL. Invasive group A streptococcal disease. Infect Agents Dis 1996; 5: 157-166.

3. Wessels MR. Group A streptococcal disease in the 1990s: new clues to pathogenesis. Int $J$ Infect Dis 1996; 1: 107-114.

4. Ferrieri P. Microbiological features of current virulent strains of group A streptococci. Pediatr Infect Dis $J 1991 ; 10$ (10 Suppl): S20-S24.

5. Martin PR, Hoiby EA. Streptococcal serogroup A epidemic in Norway 1987-1988. Scand J Infect Dis 1990; 22: 421-429.

6. Johnson DR, Stevens DL, Kaplan EL. Epidemiologic analysis of group A streptococcal serotypes associated with severe systemic infections, rheumatic fever, or uncomplicated pharyngitis. J Infect Dis 1992; 166: 374-382.

7. Chaussee MS, Liu J, Stevens DL, Ferretti JJ. Genetic and phenotypic diversity among isolates of Streptococcus pyogenes from invasive infections. J Infect Dis 1996; 173: 901-908.

8. Greco R, De Martino L, Donnarumma G, Conte MP, Seganti L, Valenti P. Invasion of cultured human cells by Streptococcus pyogenes. Res Microbiol 1995; 146: 551-560.

9. LaPenta D, Rubens C, Chi E, Cleary PP. Group A streptococci efficiently invade human respiratory epithelial cells. Proc Natl Acad Sci USA 1994; 91: 12115-12119.

10. Österlund A, Engstrand L. Intracellular penetration and survival of Streptococcus pyogenes in respiratory epithelial cells in vitro. Acta Otolaryngol (Stockh) 1995; 115: 685-688.

11. Molinari G, Talay SR, Valentin-Weigand P, Rohde M, Chhatwal GS. The fibronectin-binding protein of Streptococcus pyogenes, $\mathrm{SfbI}$, is involved in the internalization of group A streptococci by epithelial cells. Infect Immun 1997; 65: 1357-1363.

12. Carapetis J, Robins-Browne RM, Martin D, Shelby-James T, Hogg G. Increasing severity of invasive group A streptococcal disease in Australia: clinical and molecular epidemiological features and identification of a new virulent M-nontypable clone. Clin Infect Dis 1995; 21: 1220-1227.

13. Wessels MR, Moses AE, Goldberg JB, DiCesare TJ. Hyaluronic acid capsule is a virulence factor for mucoid group A streptococci. Proc Natl Acad Sci USA 1991; 88: 8317-8321.

14. Wessels MR, Bronze MS. Critical role of the group A streptococcal capsule in pharyngeal colonization and infection in mice. Proc Natl Acad Sci USA 1994; 91: 12238-12242.

15. Caparon MG, Stephens DS, Olsén A, Scott JR. Role of M protein in adherence of group A streptococci. Infect Immun 1991; 59: $1811-1817$.

16. Norgren M, Caparon MG, Scott JR. A method for allelic replacement that uses the conjugative transposon $\operatorname{Tn} 916$ : deletion of the emm6.1 allele in Streptococcus pyogenes JRS4. Infect Immun 1989; 57: 3846-3850.

17. Robins-Browne RM, Bennett-Wood V. Quantitative assessment of the ability of Escherichia coli to invade cultured animal cells. Microb Pathog 1992; 12: 159-164.

18. Tran P-OT, Johnson DR, Kaplan EL. The presence of $M$ protein in nontypeable group A streptococci upper respiratory tract isolates from southeast Asia. J Infect Dis 1994; 169; $658-661$.

19. Hollingshead SK, Simecka JW, Michalek SM. Role of M protein in pharyngeal colonization by group A streptococci in rats. Infect Immun 1993; 61: 2277-2283.

20. Wang J-R, Stinson MW. M protein mediates streptococcal adhesion to HEp-2 cells. Infect Immun 1994; 62: 442-448.

21. Valentin-Weigand P, Chhatwal GS. Correlation of epithelial cell invasiveness of group B streptococci with clinical source of isolation. Microb Pathog 1995; 19: 83-91.

22. Fischetti VA. Streptococcal M protein: molecular design and biological behavior. Clin Microbiol Rev 1989; 2: 285-314.

23. Schwartz B, Facklam RR, Breiman RF. Changing epidemiology of group A streptococcal infection in the USA. Lancet 1990; 336: $1167-1171$.

24. Liu S, Courtney HS, Bessen DE, Hasty DL, Dale JB. M protein expression is not required for resistance to phagocytosis of type 18 group A streptococci. In: Horaud T, Bouvet A, Leclercq R, de Montclos H, Sicard M (eds) Streptococci and the host. New York, Plenum. Adv Exp Med Biol 1997; 418: $725-727$. 